The Scottish Inter-collegiate Guideline Network guideline 47. Preventing dental caries in children at high caries risk: targeted prevention of dental caries in the permanent teeth of 6-16 year olds presenting for dental care

\title{
Nigel Pitts
}

\section{Director, Dental Health Services Research Unit, Dundee, Scotland}

An increasing number of dentists in a variety of countries ask about the Scottish Inter-collegiate Guideline Network (SIGN) caries guideline and the background issues that led to its publication. I have set out the details of SIGN and its first guideline below as a structured editorial, in the form of questions and answers.

Evidence-Based Dentistry (2002) 3, 93-95. doi:10.1038/sj.ebd.6400137

What is it?

This document is an evidence-based clinical practice guideline. There is considerable confusion about the generic term 'guideline', so it may be helpful to cite the SIGN definition for clarity, "Clinical guidelines are systematically developed statements which assist in decision-making about appropriate healthcare for specific clinical conditions."

Systematic reviews of methods for moving research into clinical practice ${ }^{1}$ show that a greater degree of success can be achieved when guidelines are implemented and 'owned' locally. Unfortunately the resources and skills required to undertake this type of task to a high standard and with multiprofessional input limit the ability of many groups of local clinicians to develop such aids to clinical practice. The solution to this problem adopted in Scotland is that SIGN develops national guidelines to a standardised methodology in order to maximise validity and then these national guidelines are critically reviewed and adapted at a local level for local implementation.

What is it not?

There has been some confusion about other valuable evidence-based initiatives related to dental caries, such as the 2001 National Institutes for Health (NIH) Consensus Development Conference "The Diagnosis and Management of Dental Caries Throughout Life" ${ }^{\prime 2}$ and the systematic reviews undertaken for that conference. ${ }^{3}$ The
SIGN guideline was prepared over the period 1997-1999 by a multiprofessional group given a very specific topic relating only to the permanent teeth of children within a defined age group attending for dental care at the National Health Service in Scotland. The systematic review component of developing the guideline is important, but is only one of the eight steps involved in the process (see below). On the other hand, the NIH conference and its reviews had a much broader series of questions to address with different and wider parameters, set for different purposes, and it was undertaken somewhat later.

It is worth learning from their application in medical practice, that such clinical guidelines of the SIGN type are not,

1. "an empty marketing slogan",

2. "cookbook" medicine (or dentistry),

3. "the end of clinical freedom", or

4. about "change for change's sake".

They are a tool to be used by the clinician to help inform his or her individual decisions about the care of a specific patient. 


\section{Why was it produced?}

All candidate topics for SIGN guidelines must be proposed on the basis of evidence that there is: substantial variation in clinical practice, a reasonable body of high-quality evidence applying specifically to the topic and a significant burden of disease. In this case there were additional concerns from dentists that they were confused trying to judge what was an effective treatment in this area, and national statistics showing an increase in apparently inappropriate practice.

The purpose of these (and all) SIGN guidelines is to promote effective healthcare by reinforcing good clinical practice. In addition, the aim is to promote change in professional practice where this does not comply with current best practice.

\section{Who was responsible for its production?}

SIGN works as a professionally-led group of nominees from the royal colleges and their faculties in Scotland, together with other interested parties. The work is supported financially by the National Health Service in Scotland. SIGN is a broad network of representatives from royal medical colleges, dentistry, nursing, pharmacy, professions allied to medicine, public health and patients. The multidisciplinary participation in the Council, guideline development groups and methodology groups is seen as essential to ensure: proper evaluation and interpretation of specialty-specific evidence; relevance to the realities of everyday practice; and ownership and co-operation of all stakeholder groups.

The guideline development group for SIGN 47 comprised 11 individuals drawn from paediatric dentistry, general dental practice, community dental practice, dental public health, general medical practice and health services research. Patient input was achieved via an open national meeting of the draft guideline. The author had the privilege to chair the group (members listed in full in the guideline at www.sign.ac.uk) and would like to explicitly acknowledge them all for their invaluable input.

\section{How was it produced?}

The SIGN methodology (as it existed at that time) was observed. This was undertaken using the following eight steps:

1. Selection of guideline topic

2. Identification of a suitable guideline development group

3. Systematic literature review

4. Formation and grading of recommendations

5. Consultation and peer review

6. Publication and dissemination

7. Steps to promote implementation

8. Review (on-going)

The European AGREE system for appraising guideline quality (see www.agreecollaboration.org) has demonstrated that it produces clear, valid and robust guidelines suitable for the target users.

\section{What are the key recommendations? Format of recommendations:}

An essential feature of the SIGN system is that recommendations made are explicitly linked to the strength of the evidence identified in the systematic review undertaken to answer the group's questions. The grading of evidence is by a hierarchy based on the research design and on the quality of studies.

The guideline development group used itsjudgement to make recommendations based on factors including: the volume of evidence, the consistency of evidence, its ability to be generalised, clinical impact and resource implications.

Recommendations were judged to be Grade A [at least one randomised controlled trial (RCT) as part of a body of literature of overall good quality and consistency addressing the specific recommendation], Grade B (well-conducted clinical studies, but no RCT on the topic of recommendation), Grade C (evidence obtained from expert-committee reports or opinions and/or experiences of well-respected authorities; an absence of directly applicable studies of good quality), or Good Practice Points (recommended best practice based on the clinical experience of the guideline development group).

Recommendations were made in the following fields:

Primary prevention of dental caries

- Making an explicit caries risk assessment (B)

Behaviour modification in children at high risk of caries

- Dentalhealth education at the chairside (A)

- Brushing teeth with fluoride toothpaste of 1000 ppm (A)

- Restriction of sugary food and drink (C)

- Encouragement to use non-sugar sweeteners (B) and sugar-free gum (B)

- Clinicians should use sugar-free medicines when appropriate (B)

Tooth protection in children at high risk of caries

- Sealants should be applied and maintained in pits and fissures of children at high risk of caries (A)

- Condition of sealants to be reviewed at each check-up (B) and glass ionomer sealants to be used only when resin sealantsare unsuitable (B)

- Daily fluoride tablets to be considered for children at high risk of decay (B)

- Fluoride varnish may be applied every 4-6 months to the teeth of children at high risk of caries (B)

- Chlorhexidine should be considered as an option for preventing caries (B)

Secondary and tertiary prevention of dental caries

Diagnosis of dental caries

- Bitewing radiographs are recommended as an essential adjunct to the patient's first examination (A)

- The frequency of further radiographic examination should be determined by an assessment of the patient's caries risk (B) 
Management of carious lesions occlusal

- If only part of the fissure system is involved with small-moderate dentine lesions with moderate extension, the treatment of choice is a composite sealant restoration (B)

- If caries extends clinically into dentine, then carious dentine should be removed and the tooth restored (B)

- Dental amalgam is an effective filling material, which remains the treatment of choice in many clinical situations. There is no evidence that amalgam restorations are hazardous to general health (B)

Management of carious lesions approximal

- Preventive care, eg, topical fluoride varnish, rather than operative care is recommended when approximal caries is confined (radiographically or visually) to enamel (A)

- In an approximal lesion requiring restoration, a conventional Class II restoration should be placed in preference to a tunnel preparation (B)

\section{Re-restoration}

- The diagnosis of secondary caries is extremely difficult and clear evidence of involvement of active dis- ease should be ascertained before replacing a restoration $(\mathrm{B})$

Access to the guideline and features to promote use

The Guideline exists in three forms. It is available as the Quick Reference Guide (a 2-page user-friendly summary; the full SIGN guideline (42 pages); and as the full reference document. The latter details the process, membership, any conflicts of interest, peer review, comments from the Editorial Board and the national meeting, and the responses of the group to these comments. In addition to the recommendations, the guideline document also includes tools to promote local use, audit and research.

In physical terms the document is provided as a printed version (mailed to every dentist in Scotland), a CD-ROM version, and there is WWW access with associated downloadable Adobe Acrobat (.pdf) files (please refer to www.sign.ac.uk). SIGN can be contacted by e-mailed on sign@rcpe.ac.uk.

\section{Review of the guideline}

The guideline will be updated soon, with a consideration of new literature and comments received by SIGN. Details of SIGN methodology, which has also been reviewed and which has evolved over the last 3 years, is available via the WWW, or as a handbook called SIGN 50. This contains details of a refined version of the methodology for assessing papers and grading recommendations built on the experience of the first 50 SIGN guidelines.

\section{The future}

Having undertaken this guideline and one other (Third Molar Teeth; guideline no. 43), the SIGN Council has recently agreed (via a process of judging competitive applications) that caries in the preschool child will be the subject of a forthcoming review and guideline. In addition, orofacial cancer has been identified as a potential topic to be adopted when funds allow.

1. Effective Health Care Bulletin. Getting Evidence into Practice. NHS Centre for Reviews and Dissemination February 1999 volume 5 number 1 ISSN: 0965-0288 (www.york.ac.uk/inst/crd/ehc51.htm)

2. National Institutes for Health. NIH Consensus Statement: Diagnosis and Management of Dental Caries Throughout Life. Vol. 18, No.1. 26-28 March, 2001. Bethesda: National Institutes of Health, Office of the Director

3. National Institutes for Health. Evidence Report/Technology Assessment No. 36, Diagnosis and Management of Dental Caries (AHRQ Publication No. 01-E056). www.ahrq.gov/clinic/epcsums/ dentsumm.htm 